\title{
Quantitative permeability imaging of plant tissues
}

\author{
Timur A. Sibgatullin • Frank J. Vergeldt • \\ Edo Gerkema · Henk Van As
}

Received: 4 August 2009/Revised: 14 October 2009/Accepted: 19 October 2009/Published online: 17 November 2009

(C) The Author(s) 2009. This article is published with open access at Springerlink.com

\begin{abstract}
A method for mapping tissue permeability based on time-dependent diffusion measurements is presented. A pulsed field gradient sequence to measure the diffusion encoding time dependence of the diffusion coefficients based on the detection of stimulated spin echoes to enable long diffusion times is combined with a turbo spin echo sequence for fast NMR imaging (MRI). A fitting function is suggested to describe the time dependence of the apparent diffusion constant in porous (bio-)materials, even if the time range of the apparent diffusion coefficient is limited due to relaxation of the magnetization. The method is demonstrated by characterizing anisotropic cell dimensions and permeability on a subpixel level of different tissues of a carrot (Daucus carota) taproot in the radial and axial directions.
\end{abstract}

Keywords Plant tissue permeability · Cell sizes ·

Restricted diffusion - Time-dependent diffusion coefficient

The more you see: spectroscopy in molecular biophysics.

Electronic supplementary material The online version of this article (doi:10.1007/s00249-009-0559-1) contains supplementary material, which is available to authorized users.

T. A. Sibgatullin · F. J. Vergeldt · E. Gerkema · H. Van As ( $\square)$ Laboratory of Biophysics and Wageningen NMR Centre, Wageningen University, Dreijenlaan 3,

6703 HA Wageningen, The Netherlands

e-mail: Henk.vanAs@wur.nl

URL: www.bip.wur.nl; www.wnmrc.nl

T. A. Sibgatullin

Kazan Institute of Biochemistry and Biophysics,

Kazan Scientific Center, Russian Academy of Sciences,

Lobachevsky Street 2, 420111 Kazan, Russia

\section{Introduction}

Water diffusing in a confined space, such as cells and pores, has proven to be a very informative internal probe for nuclear magnetic resonance (NMR) in many systems. The diffusion coefficient and related properties such as the transverse relaxation time $T_{2}$ no longer reflect the molecular or bulk parameters, such as the diffusion coefficient $D_{0}$ and $T_{2, \text { bulk }}$ but become dependent on the size, shape, and permeability of the enclosed space (Grebenkov 2007). For this reason the measurement of the apparent diffusion coefficient (ADC) by pulsed field gradient (PFG) NMR (Stejskal and Tanner 1965), eventually in combination with magnetic resonance imaging (MRI), has found a wide variety of applications in biomedicine (Norris 2001), biology, material sciences, geology, and many other scientific disciplines (Codd and Seymour 2008; Sen 2004).

To extract information on size and membrane permeability of cells, ADCs have to be measured as a function of the diffusion encoding or observation time $t_{\mathrm{d}}$ (Latour et al. 1994; Mitra et al. 1993; Sen 2003; Tanner 1978). By varying $t_{\mathrm{d}}$, the distance over which the spins can diffuse freely and the extent to which they can pass across the cell membrane, which restricts the diffusion process, can be observed. The probability of passing across the membrane is a direct measure of the water permeability of the surrounding membrane (Regan and Kuchel 2000). At longer values of $t_{\mathrm{d}}$, water molecules can even travel from cell to cell, and information on extracellular tortuosity or tissue permeability becomes available.

Alternative methods of obtaining membrane permeability from PFG NMR are based on the analysis of diffusional exchange (Kärger 1971; Kärger et al. 1988; Pfeuffer et al. 1998b; Waldeck et al. 1995). On the basis of this, Pfeuffer et al. (1998a) performed in vivo quantitative 
mapping of diffusional exchange in rat brain tissue. Paran et al. (2004) demonstrated in vivo quantitative mapping of breast tumors. But the analysis is complicated due to the compartmentalization of the tissue with a large contribution of the extracellular compartment.

Plant cell sizes are large in comparison to animal tissue cells with diameters in the range of 10-100 $\mu \mathrm{m}$ and even more. In this case it takes more time for water molecules to probe the dimensions of the cell by diffusion. It was demonstrated that $T_{2}$ of water confined in plant cells is sensitive to variances in cell size and permeability of cell membranes (van der Weerd et al. 2002a). However the contribution of these two factors can not be separated without prior knowledge of one of them. PFG NMR offers an alternative approach to determine the cell size and permeability of plant tissue (Anisimov et al. 1998). The time dependence of the diffusion coefficient differs from tissue to tissue within plants and shows anisotropy in relation to the direction of growth (Boujraf et al. 2001; Kuchenbrod et al. 1995; Scheenen et al. 2001). However, in PFG NMR experiments, diffusion time is limited by the longitudinal relaxation time $T_{1}$ or $T_{2}$, based on the chosen approach. Usually it is not possible to measure the long time limit of the ADC. As a result, it is difficult to quantify structural parameters.

Valiullin et al. developed a method to overcome these complications (Valiullin and Skirda 2001; Valiullin et al. 1997). A fitting function was derived on the basis of theoretical work to describe the time dependence of the ADC. It enables simultaneous determination of cell (or pore) size and permeability (interconnectivity or tortuosity) of the system even when the long time limit of the diffusion constant can not be measured experimentally. On the basis of this approach, cell sizes and permeability of different systems such as root segments of maize (Anisimov et al. 1998), peanuts (Zakhartchenko et al. 1998), single-celled algae (Cho et al. 2003), yeast cells (Hong et al. 2004; Suh et al. 2003), apple tissue (Sibgatullin et al. 2007), and soaked rice (Hong et al. 2009) have been characterized.

So far this approach for tissue characterization has only been demonstrated in nonimaging NMR experiments. In this paper, we extend the approach with MR imaging. This method for quantitative permeability mapping is used to characterize the different tissues in the taproot of carrot (Daucus carota). Since MR imaging involves the use of gradients that can affect the PFG approach, the influence of these imaging gradients on the calculation of diffusion constants is discussed and corrected for.

\section{Theory}

The diffusion coefficient $D_{0}$ of bulk water is independent of $t_{\mathrm{d}}$. However, when Brownian motion is restricted by an impermeable wall, the detected diffusion coefficient becomes dependent on $t_{\mathrm{d}}$ and is referred to as ADC or $D\left(t_{\mathrm{d}}\right)$. In cases of fully restricted diffusion, the displacement of molecules can not exceed the distance $R$ between enclosing barriers. For one-dimensional diffusion at the limit of the long diffusion time $\left(t_{\mathrm{d}} \gg R^{2} / D_{0}\right)$, the ADC becomes inversely proportional to $t_{\mathrm{d}}$ according to Einstein's equation

$D\left(t_{\mathrm{d}}\right)=\frac{\left\langle r^{2}\right\rangle}{2 t_{\mathrm{d}}}$

where the mean square displacement $\left\langle r^{2}\right\rangle$ is constant and proportional to $R^{2}$ with the coefficient dependent on the geometry of the barriers.

For partially restricted diffusion, Valiullin et al. (1997) suggested a renormalization of $D\left(t_{\mathrm{d}}\right)$ that eliminates the effect of permeability of the barriers or interconnected pores

$D_{\text {eff } 1}\left(t_{\mathrm{d}}\right)=D_{0} \frac{D\left(t_{\mathrm{d}}\right)-D_{\infty}}{D_{0}-D_{\infty}}$

with $D_{0}$ as the bulk diffusion coefficient at $t_{\mathrm{d}}=0$ and $D_{\infty}$ in the long diffusion time limit $\left(t_{\mathrm{d}}=\infty\right)$. $D_{\text {eff } 1}\left(t_{\mathrm{d}}\right)$ behaves in much the same way as for the case of fully restricted diffusion. This makes it possible to apply Eq. 1 and estimate the size of restrictions for intermediate or long diffusion times based on the size of the restrictions. This approach was proven theoretically (Valiullin and Skirda 2001) and validated with simulated and experimental data (Valiullin and Skirda 2001; Valiullin et al. 1997; Zakhartchenko et al. 1998).

In tissues with large cells, the long diffusion time limit $D_{\infty}$ is often experimentally unavailable due to relaxation of the magnetization. For this situation, a second renormalization is used to describe the time-dependent ADC of a liquid with an infinite diffusion constant $\left(D_{0}=\infty\right)$

$D_{\text {eff } 2}\left(t_{\mathrm{d}}\right)=\frac{D_{0} D_{\text {eff } 1}\left(t_{\mathrm{d}}\right)}{D_{0}-D_{\text {eff } 1}\left(t_{\mathrm{d}}\right)}$

This equation enlarges the range of diffusion times where dependence in Eq. 1 is observed to diffusion times that are shorter.

For a system with semipermeable barriers, Eqs. 2 and 3 can be used to express the measured $D\left(t_{\mathrm{d}}\right)$ as a function of the bulk diffusion coefficient of the liquid $D_{0}$, the long time limit of ADC $D_{\infty}$, and through $D_{\text {eff } 2}\left(t_{\mathrm{d}}\right)$

$D\left(t_{\mathrm{d}}\right)=D_{\text {eff } 2} \frac{\left(D_{0}-D_{\infty}\right)}{\left(D_{\text {eff } 2}+D_{0}\right)}+D_{\infty}$

Equation 4 can be used to fit the behavior of the ADC in the range of intermediate diffusion times $\left(t_{\mathrm{d}} \sim R^{2} / D_{0}\right)$ and long diffusion time limits $\left(t_{\mathrm{d}} \gg R^{2} / D_{0}\right)$. It enables the 
simultaneous determination of $D_{0}, D_{\infty}$, and the characteristic size of restrictions $R$.

$D_{\infty}$ can now be interpreted in terms of tortuosity (commonly used for porous structures) or in terms of permeability, which is more suitable for a system with semipermeable membranes. For plant tissue, the amount of extracellular water (apoplast water) is much smaller then the amount of intracellular water. The main volume of the cell in mature parenchyma is occupied by the vacuole. As a result, plant tissue can be roughly approximated as a system of closely packed water containers separated by semipermeable membranes. In our case, a simplified model of parallel semipermeable planes (Crick 1970) was used to calculate the value of permeability $P$

$\frac{1}{D_{\infty}}=\frac{1}{D_{0}}+\frac{1}{P \cdot R}$

with $R$ as the distance between the membranes. Correction coefficients were applied to take into account the actual geometry of the cells.

\section{Materials and methods}

\section{MR imaging}

All measurements were performed on a $3 \mathrm{~T}(128 \mathrm{MHz}$ for protons) MRI system (Bruker, Karlsruhe, Germany), consisting of an Avance console, a superconducting magnet with a $50 \mathrm{~cm}$ vertical free bore (Magnex, Oxford, UK), a 1 $\mathrm{T} / \mathrm{m}$ gradient coil, and a birdcage RF coil with an inner diameter of $4 \mathrm{~cm}$.

A high resolution image was obtained using a turbo spin echo (TSE) MRI sequence (Scheenen et al. 2000) with a repetition time (TR) of $5 \mathrm{~s}$, an effective spin echo time (TE) of $12.5 \mathrm{~ms}$, and a spectral bandwidth (SW) of $50 \mathrm{kHz}$. Only eight echoes were acquired in the TSE train to avoid $T_{2}$ weighting. Odd and even echoes were separately phase-encoded forming two different images to avoid Nyquist ghost's artifacts, so the turbo factor was 4 . Two acquisitions were averaged to improve image quality. The field-of-view (FOV) was $40 \times 40 \mathrm{~mm}^{2}$ with a matrix size of $512 \times 512$ resulting in an in-plane resolution of $78 \times 78 \mu \mathrm{m}^{2}$. The slice thickness was $2 \mathrm{~mm}$.

$\mathrm{T}_{2}$ mapping was done using a multi spin echo (MSE) imaging sequence (Edzes et al. 1998), a TR of $5 \mathrm{~s}$, a TE of $8.2 \mathrm{~ms}$, and an $\mathrm{SW}$ of $50 \mathrm{kHz}$. For each echo train, 128 echoes were acquired; two acquisitions were averaged to improve image quality. The FOV was $40 \times 40 \mathrm{~mm}^{2}$ with a matrix size of $256 \times 256$ resulting in an in-plane resolution of $156 \times 156 \mu \mathrm{m}^{2}$. The slice thickness was $2 \mathrm{~mm}$.

Diffusion mapping was done using a pulsed field gradient-stimulated echo PFG-STE sequence for diffusion measurements combined with a TSE sequence for fast imaging (PFG-STE-TSE, Fig. 1) (Scheenen et al. 2001). Data were acquired with a TR of $5 \mathrm{~s}$ and an effective TE of $5.5 \mathrm{~ms}$. Cross-sectional images were obtained with a FOV of $40 \times 40 \mathrm{~mm}^{2}$ and a $64 \times 64$ matrix resulting in an inplane spatial resolution of $625 \times 625 \mu \mathrm{m}^{2}$ and a slice thickness of $3 \mathrm{~mm}$. The turbo factor was 4 , while eight echoes per scan were acquired in line with the high resolution TSE measurements. A crusher gradient of 0.05 $\mathrm{T} / \mathrm{m}$ with duration of $2 \mathrm{~ms}$ was used to suppress spurious echoes. A pair of spoiler gradient pulses of $0.02 \mathrm{~T} / \mathrm{m}$ with duration $1 \mathrm{~ms}$ was used to crush the FID formed by the third $90^{\circ} \mathrm{RF}$ pulse.

Diffusion constants were determined in two directions: (1) axial-along the root, in the slice-encoding direction of the imaging experiment, and (2) in plane or radial-across the root, along the readout direction of the imaging experiment. The carrot taproot was placed along the field of the magnet. The position of the sample was kept the same for all experiments.

The diffusion time $t_{\mathrm{d}}$ was varied in nine steps from $15 \mathrm{~ms}$ up to $1 \mathrm{~s}$. For each value of $t_{\mathrm{d}}$, eight measurements were performed with linearly increasing diffusion encoding gradients $G_{\text {diff }}$ with duration $\delta_{\text {diff }}$ of 3 ms. The maximum $b$ value was kept constant for all diffusion times to provide sufficient attenuation of the signal

$\ln [A(b) / A(0)]=-\gamma^{2} \delta^{2} G_{\mathrm{diff}}^{2} t_{\mathrm{d}} D=-b D$

where $t_{\mathrm{d}}=\Delta-\delta / 3$ (Fig. 1). For short diffusion times, eight averages were acquired; for long diffusion times, 16 averages were acquired to improve the signal-to-noise ratio (SNR).

The $b$ values were corrected for the presence of other gradients that were weighted for diffusion next to the diffusion encoding gradients (Neeman et al. 1990). The

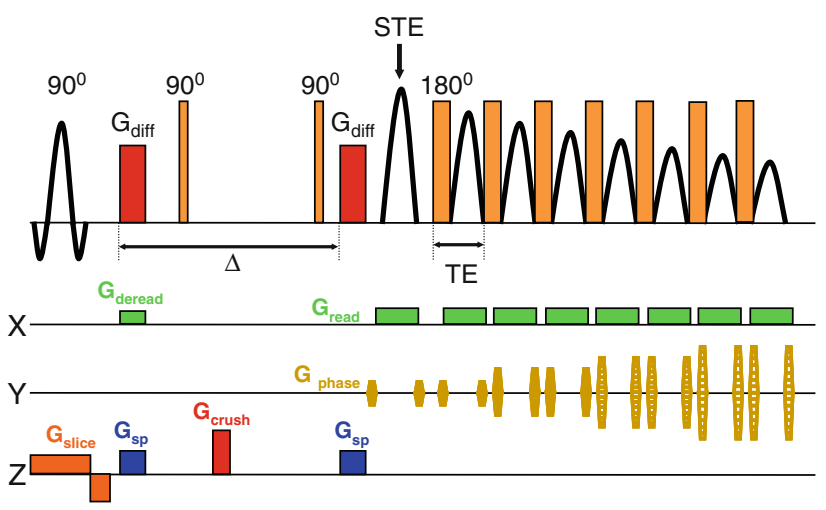

Fig. 1 The pulsed field gradient-stimulated spin echo-turbo spin echo (PFG-STE-TSE) sequence. Diffusion encoding gradients $G_{\text {diff }}$ are directed either along the slice-encoding direction for axial diffusion encoding, or along the readout direction for in-plane diffusion measurements. The stimulated echo is indicated with STE 
gradients taken into account are (1) the spoiler gradients $G_{\text {sp }}$ with duration $\delta_{\text {sp }}$, (2) the dephasing readout gradient $G_{\text {deread }}$ of duration $\delta_{\text {deread }}$, with product $\delta_{\text {deread }} G_{\text {deread }}$, which is equal to half the product of the readout gradient and the acquisition duration $t_{\mathrm{aq}} G_{\mathrm{read}}$, and (3) the diffusion gradients themselves $G_{\text {diff }}$ with duration $\delta_{\text {diff. }}$ The resulting gradient is determined as a vector sum

$\delta \vec{G}=\delta_{\text {sp }} \vec{G}_{\text {sp }}+\delta_{\text {deread }} \vec{G}_{\text {deread }}+\delta_{\text {diff }} \vec{G}_{\text {diff }}$

According to the orientation of the gradients, the resulting $b$ values were calculated as follows: for diffusion experiments in the in-plane direction

$b\left(G_{\text {diff }}\right)=\gamma^{2}\left(\delta_{\text {diff }} G_{\text {diff }}+\delta_{\text {deread }} G_{\text {deread }}\right)^{2} t_{d}+\gamma^{2}\left(\delta_{\text {sp }} G_{\text {sp }}\right)^{2} t_{d}$

and for diffusion experiments in the axial direction

$b\left(G_{\text {diff }}\right)=\gamma^{2}\left(\delta_{\text {diff }} G_{\text {diff }}+\delta_{\text {sp }} G_{\mathrm{sp}}\right)^{2} t_{d}+\gamma^{2}\left(\delta_{\text {deread }} G_{\text {deread }}\right)^{2} t_{\mathrm{d}}$

The most important role is played by the gradients that are collinear to the direction of diffusion measurement (for axial-the spoiler, for in plane-the frequency encoding gradient). Ignoring them in calculations of $b$ values leads to the nonlinear distortion of the $b$ axis and an overestimation of the ADC. Gradients perpendicular to the direction of the applied diffusion gradient only cause a linear shift of the $b$ axis, which leads to underestimation of the initial amplitude of the signal attenuation plot but does not influence the calculations of the diffusion constants.

\section{Plant material}

A well developed fully matured taproot of carrot (Daucus carota) was bought in a local supermarket. A $3 \mathrm{~cm}$ segment was cut out from the central part of the root and covered with parafilm to avoid evaporation. All experiments were performed at room temperature $\left(20 \pm 1^{\circ} \mathrm{C}\right)$.

\section{Data analysis}

All ADCs were determined from the initial slope of the signal attenuation plot (SAP) as described in Eq. 6 with $b$ values calculated as described by Eqs. 6 and 7. The time dependence of the ADCs was analyzed in two ways: (1) fitting of $D\left(t_{\mathrm{d}}\right)$ using Eqs. 1-5 on a pixel-by-pixel basis, and (2) analysis of $D\left(t_{\mathrm{d}}\right)$ in regions of interest (ROI), where ADCs were determined from the slope of the total SAP of this region. All data handling and analysis were performed with home-built software written in IDL (RSI, Boulder, CO), except for the analysis using Eqs. 1-5 of $D\left(t_{\mathrm{d}}\right)$ from the ROIs, which was done in MATLAB (The MathWorks, Natick, MA)

\section{Results}

The high resolution TSE image reveals detailed anatomy of carrot taproot (Fig. 2). The contrast between tissues is not only caused by differences in water content, but also by loss of magnetization due to $T_{2}$ weighting (the effective TE was $12.5 \mathrm{~ms}$ ) and by diffusion weighting, as the image encoding gradients act simultaneously as diffusion encoding gradients.

The same detailed structure is found in the parameter maps derived from the $T_{2}$ experiment, although with a lower spatial resolution (Fig. 3). For each pixel, the echo train was fitted to a mono-exponential decay function resulting in an amplitude map representing the proton density or water content (Fig. 3a) and a $T_{2}$ map (Fig. 3b). Some regions of the phloem tissue (section 4 in Fig. 4a) and the air spaces in the central cylinder of the xylem tissue (section 2 in Fig. 4a) show poorly hydrated areas.

At short diffusion times $\left(t_{\mathrm{d}}=15 \mathrm{~ms}\right.$, Fig. 5a, c), hardly any difference can be found in the amplitude images of different gradient steps compared to the proton density map (Fig. 3a). When $t_{\mathrm{d}}$ becomes longer, the images become more and more $T_{1}$ weighted due to the increasing storage period of the magnetization along the main magnetic field between the second and third $90^{\circ} \mathrm{RF}$ pulse, which results in extra spin-lattice relaxation (Fig. 5b, d). As a result, some pixels of the phloem region (section 4 in Fig. 4a) can not be analyzed correctly at long diffusion times since the intensity of the signal becomes too low due to fast $T_{1}$ relaxation. These pixels are black in the diffusion maps (Fig. 6).

At long diffusion times $\left(t_{\mathrm{d}}=1 \mathrm{~s}\right)$, diffusion weighting in the axial direction results in clearly observable contrast of the internal ring of the cambium (section 3 in Fig. 4a) of the carrot root (Fig. 5d). This results from restricted diffusion and differences in permeability and demonstrates the clear time dependence of the ADC in this region (Fig. 6). This dependency differs from tissue to tissue and shows anisotropy (Fig. 4b, c).

Tissues were differentiated on the basis of contrast in one of the measured parameter maps. Cambium (section 3 in Fig. 4a) was selected as the bright ring in the amplitude image measured by the in-plane diffusion experiment with maximum gradient strength at $t_{\mathrm{d}}=1 \mathrm{~s}$ (Fig. 4d, lowest image). Xylem tissue inside the ring of cambium was separated on the basis of the proton density map (Fig. 2a) into two regions: a region with high intensity (section 1 in Fig. 4a) and a region of low (nearly zero) intensity (section 2 in Fig. 4a). Cortex tissue (section 3 in Fig. 4a) was selected on the basis of $T_{2}$ contrast (Fig. 2b) as it was characterized by relaxation times much longer than that of the phloem and epidermis (section 4 in Fig. 4a). 


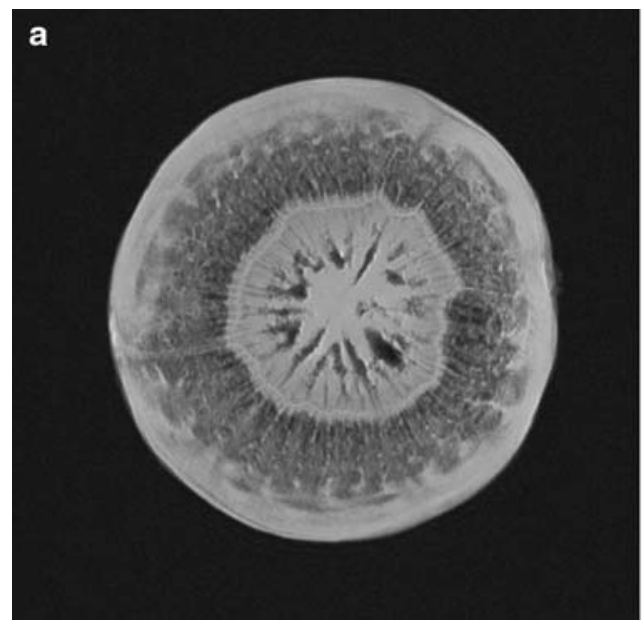

Fig. 2 a High resolution turbo spin echo image of carrot taproot. Experimental parameters: TSE sequence, in-plane matrix size $512 \times 512$, spatial resolution $78 \times 78 \times 2,000 \mu^{3}$, effective echo

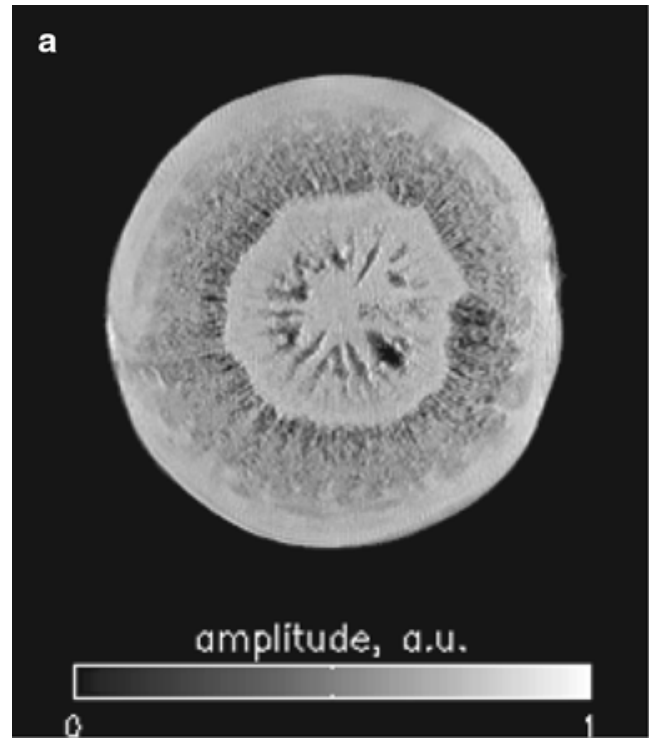

Fig. 3 a Calculated amplitude map and $\mathbf{b} T_{2}$ map as result of a multi spin echo imaging sequence. Experimental parameters: MSE sequence, in-plane matrix size $256 \times 256$, spatial resolution

For each ROI, the time-dependent ADC was fitted with Eq. 4. As a result, parametric maps of bulk and long-time diffusion coefficients $D_{0}$ and $D_{\infty}$ and cell size $R$ were determined (Fig. 7). On the basis of these results, the permeability $P$ per ROI was also calculated using the simple model for parallel semipermeable planes (Eq. 5).

The same procedure was performed in a pixel-by-pixel mode (Fig. 8). In this way detailed structural information over the cross section of the carrot root can be obtained. However, the uncertainty of the calculated permeability increases if the measured drop in ADC becomes comparable to the standard deviation (SD) of ADC. This situation is for instance observed for the axial diffusion (Fig. 8a,

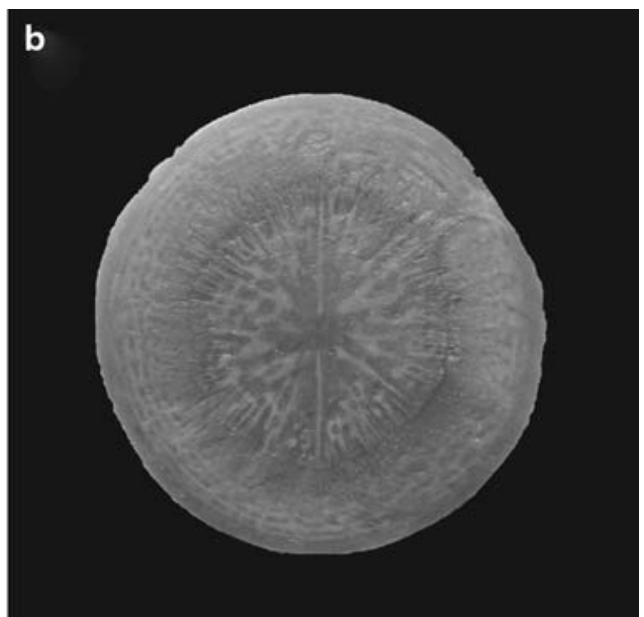

time $12.5 \mathrm{~ms}$, eight echoes in the TSE echo train, turbo factor 4 , spectral bandwidth $50 \mathrm{kHz}$, and repetition time TR $5 \mathrm{~s}$. b Photograph of carrot taproot for comparison (different taproot)

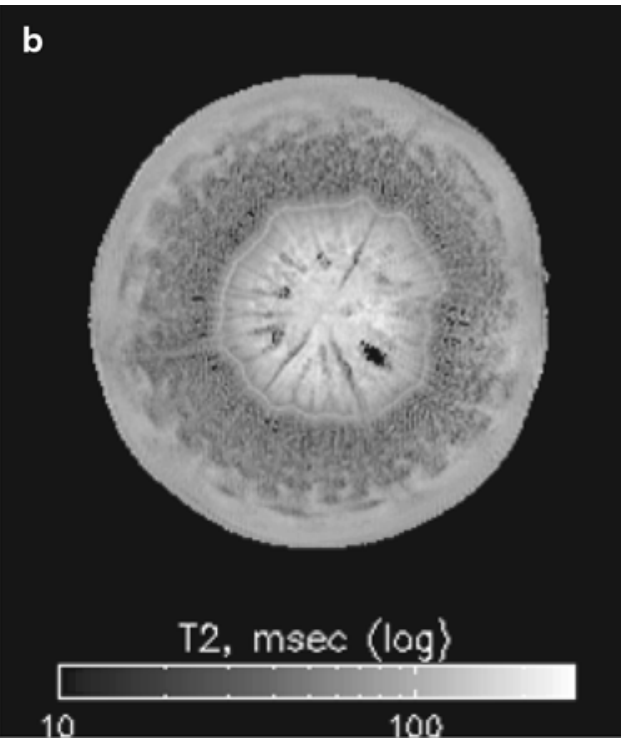

$156 \times 156 \times 2,000 \mu^{3}$, inter-echo time TE $8.2 \mathrm{~ms}, 128$ echoes in the echo train, spectral bandwidth $50 \mathrm{kHz}$, and repetition time TR $5 \mathrm{~s}$

permeability map) in several regions of the xylem tissue (sections 1 and 2 in Fig. 4a). So a main requirement of the method can be expressed with the following relation

$D_{0}-D_{\infty}>\mathrm{SD}(\mathrm{ADC})$

SD(ADC) also determines the range of $P$ and pore (cell) size $R$ as

$P \cdot R<\frac{D_{0}^{2}}{\mathrm{SD}(\mathrm{ADC})}$

since they are related to $D_{\infty}$ through Eq. 5 .

In Fig. 9, the decimal logarithm of the fitted parameters from the analysis per ROI (Fig. 7) together with their error 
Fig. 4 a Selection masks for different regions of interest: 0 a high resolution TSE image (Fig. 1) for reference, $l$ highly hydrated xylem tissue, 2 poorly hydrated xylem tissue, 3 zone of cambium cells, 4 phloem tissue and epidermis, 5 cortex. ADC as function of diffusion time for axial (b) and in-plane (c) diffusion experiments in the various regions of interest
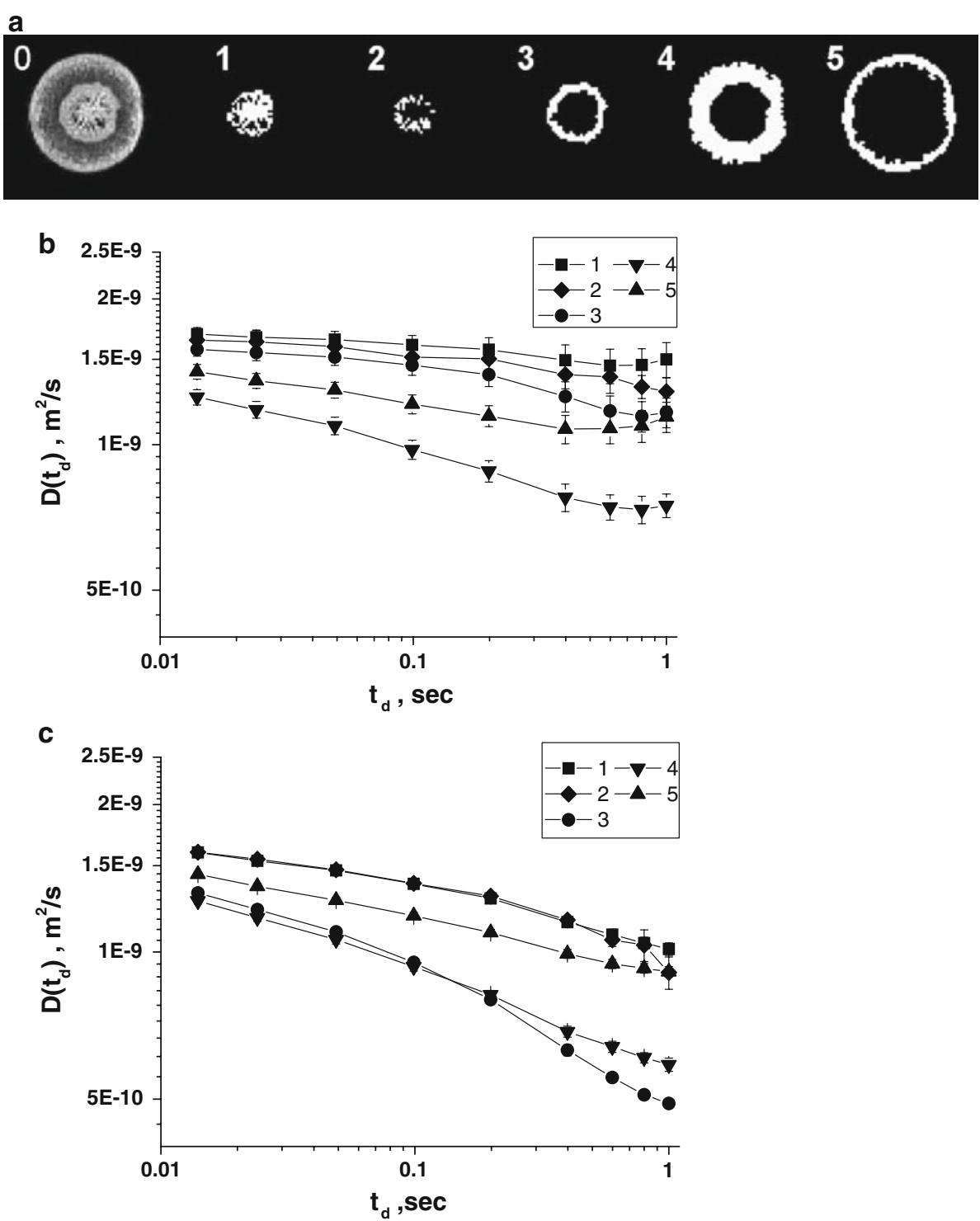

of fit are compared with the decimal logarithm of the averaged parameters from the same ROI as a result of the pixel-by-pixel approach (Fig. 8) with standard deviations of the mean. The choice to present the decimal logarithm was based on the fact that the distribution of the logarithm of the parameters per ROI was near to Gaussian.

\section{Discussion}

In the present study, a simplified model of tissue permeability was implemented to characterize diffusive transport in plants. Diffusive water transport in root tissue has been described in terms of a composite transport model (Steudle 2001). This model considers three pathways for water transport: the extracellular or apoplastic pathway, the symplastic pathway, and the transcellular pathway. The latter two pathways involve cell-to-cell transport and cannot be discriminated by present techniques. Thus cell membrane and extracellular tortuosity plays an important role in diffusive transport in plant tissue. In addition xylem conduits (vessels and tracheids) and elongated phloem cells provide for long-distance transport of water in low resistance pathways in the axial direction of a plant.

Membrane permeability in plant cells as well as in many other systems has been determined using the NMR relaxation times of intracellular water protons based on the Conlon-Outhred technique (Conlon and Outhred 1972). The disadvantage of this technique is that it requires the introduction of paramagnetic ions such as $\mathrm{Mn}^{2+}$ at nonphysiological concentrations. A noninvasive approach is based on the principle that the observed transverse relaxation time $T_{2, \mathrm{obs}}$ of water in a confined compartment such as a vacuole can be described as a function of $T_{2, \text { bulk }}$ of the 


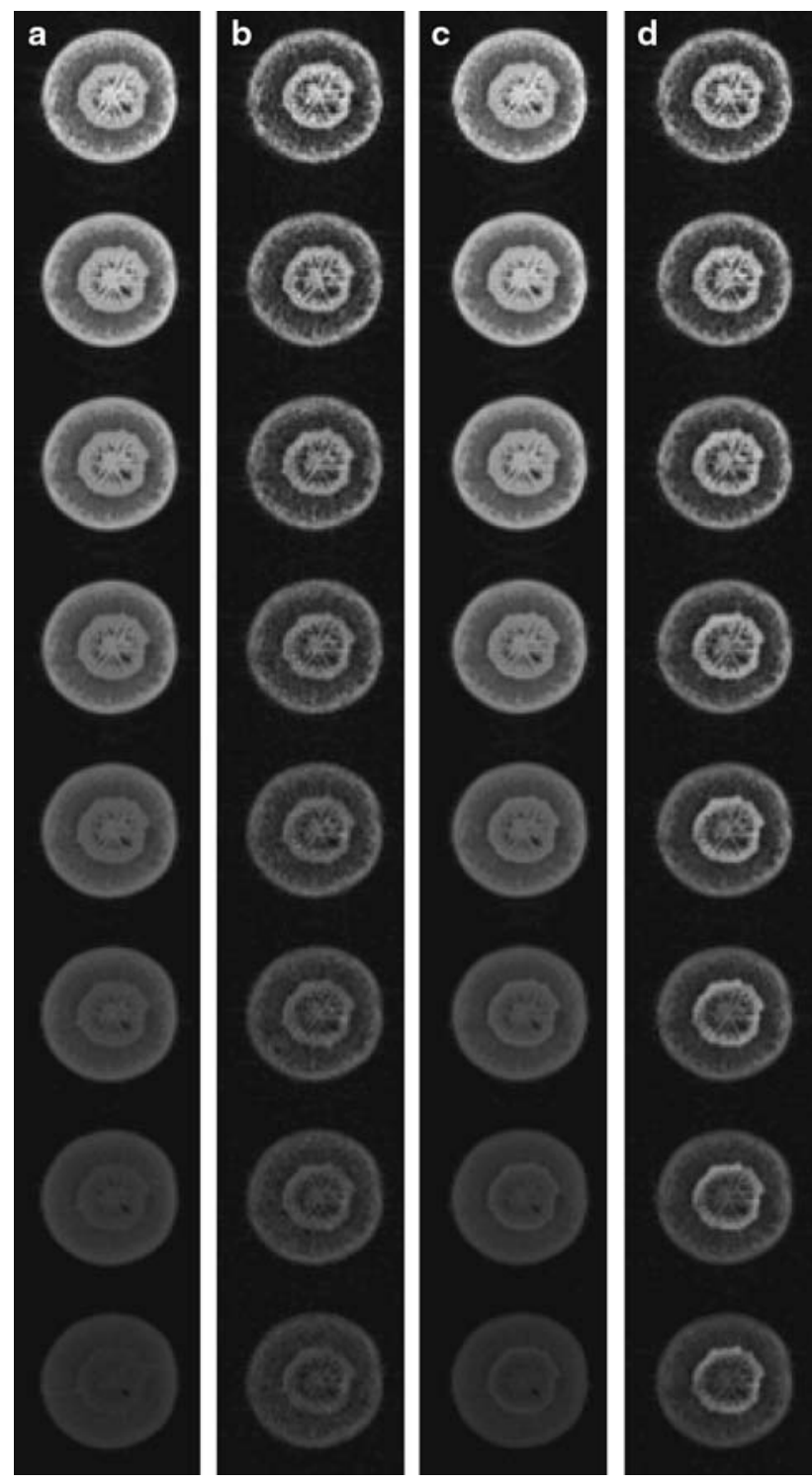

Fig. 5 Attenuation of the signal due to the diffusion at increasing $b$ values (in vertical direction from top downwards) and due to $T_{1}$ relaxation at short and long diffusion times (b compared to $\mathbf{a}$; d compared to c). Axial diffusion encoding for $\mathbf{a} t_{\mathrm{d}}=15 \mathrm{~ms}$ and $\mathbf{b}$ $t_{\mathrm{d}}=1 \mathrm{~s}$; in-plane diffusion encoding for $\mathbf{c} t_{\mathrm{d}}=15 \mathrm{~ms}$ and $\mathbf{d} t_{\mathrm{d}}=1 \mathrm{~s}$. Experimental parameters: PFG-STE-TSE sequence, in-plane matrix size $64 \times 64$, spatial resolution $625 \times 625 \times 3,000 \mu \mathrm{m}^{3}$, effective echo time $12.5 \mathrm{~ms}, 8$ echoes in the TSE echo-train, turbo factor 4 , spectral bandwidth $50 \mathrm{kHz}$, and repetition time TR $5 \mathrm{~s}$. Diffusion was encoded for diffusion gradients of duration $3 \mathrm{~ms}$ and eight different diffusion gradient strengths such that the $b$ values were the same for all diffusion encoding times

water and the probability that water molecules reach the membrane and lose magnetization at the membrane by passing across the membrane and entering a compartment with a (much) shorter relaxation time (Brownstein and Tarr 1979; van der Weerd et al. 2001). The net loss of magnetization in a vacuole therefore depends on the membrane water permeability of the tonoplast and the effective relaxation in the cytoplasm (van der Weerd et al. 2002a). As a result, the observed relaxation time depends on, in addition to $T_{2, \text { bulk }}$, the surface-to-volume ratio $S / V$ of the compartment, and the net loss of magnetism at the compartment boundary, the so-called magnetization sink strength $H$ (van der Weerd et al. 2001)

$\frac{1}{T_{2, \mathrm{obs}}}=\frac{1}{T_{2, \mathrm{bulk}}}+H \frac{S}{V}$

where $H$ is linearly related to the actual membrane permeability (van der Weerd 2002; van der Weerd et al. 2002b).

The permeability $P$ determined in this study includes the permeability of the tonoplast, plasmalemma, walls, and plasmodesmata of neighboring cells. At long observation times, cell-to-cell transport becomes visible. Therefore, $P$ is not identical to $H$ as obtained from $T_{2}$ measurements (Eq. 12) (Van As 2007). The value of $R$ as a result of the diffusion measurements can directly be used to obtain $H$ from the $T_{2}$ measurements. A complication is the effect of the geometry of the confined compartment. Equation 5 assumes plan-parallel membranes, which might be a good assumption for an array of cells in one dimension (the magnetic field gradient direction). The result of $H$ from Eq. 12 depends on the actual geometry of the compartment: for a sphere, the first term in Eq. 12 becomes $3 H / R$; for a cylindrical geometry it becomes $2 H / R$. This problem has been overcome by determining $S / V$ from $D\left(t_{\mathrm{d}}\right)$ (Van As 2007).

Sibgatullin et al. (2007) compared both approaches. By combining the results of $T_{2}$ and $D$ measurements in apple parenchyma (Granny Smith), for the vacuole $H$ was found to be around $1 \times 10^{-5} \mathrm{~m} \mathrm{~s}^{-1}$ and $R 86 \mu \mathrm{m}$. Under the assumption of parallel planes, $P$ was found to be $2.9 \times 10^{-6} \mathrm{~m} \mathrm{~s}^{-1}$. For Cox apple parenchyma cells, a tonoplast water membrane permeability of $2.5 \times$ $10^{-5} \mathrm{~m} \mathrm{~s}^{-1}$ based on the Conlon-Outhred method was previously reported (Snaar and Van As 1992). In maize roots, a higher value of $P$ around $5 \times 10^{-5} \mathrm{~m} \mathrm{~s}^{-1}$ was found (Anisimov et al. 1998). More recently values of $P$ were obtained for excised roots of normal and osmotically stressed maize and pearl millet plants; $P$ was found to be around $3 \times 10^{-5} \mathrm{~m} \mathrm{~s}^{-1}$ for both normal and stressed maize, whereas $P$ was around $9 \times 10^{-5} \mathrm{~m} \mathrm{~s}^{-1}$ for normal pearl millet plants and $3 \times 10^{-5} \mathrm{~m} \mathrm{~s}^{-1}$ for stressed plants (T.A. Sibgatullin and H. Van As, unpublished results). Ionenko et al. (2006) reported $P$ values for water in roots of maize seedlings of $3 \times 10^{-5} \mathrm{~m} \mathrm{~s}^{-1}$, which decreased by a factor of 1.7 due to water stress or $\mathrm{HgCl}_{2}$ treatment. The latter clearly demonstrates the contribution of aquaporin functioning in $P$. Up to now $H$ was found to be higher than $P$, as expected. 


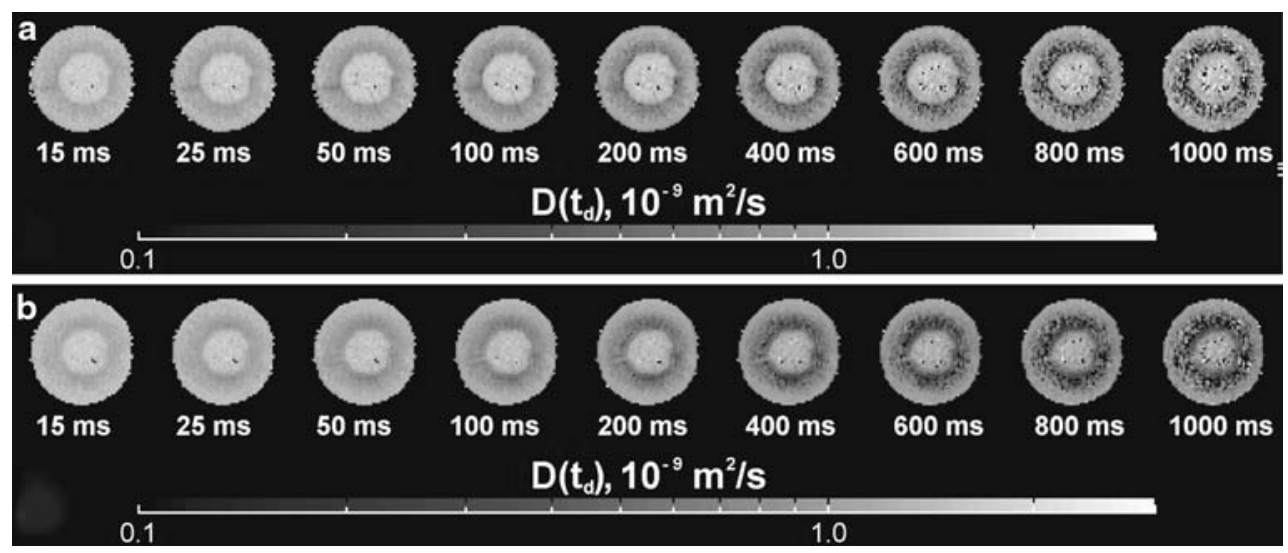

Fig. 6 Calculated ADC maps as a function of diffusion time for axial (a) and in-plane (b) diffusion experiments

In this study, time-dependent diffusion measurements for tissue characterization were extended with MR imaging. Compared to previous nonimaging studies, our method offers several advantages. First of all it enables the simultaneous characterization of different tissue types based on anatomical features recognizable in the MR images. Furthermore different NMR contrast mechanisms can be used to increase the quality of the segmentation. Finally the selection of different tissue types can be analyzed pixel-bypixel for high spatial resolution or summed together to increase SNR and obtain high quality parameter values per tissue type. This approach for SNR enhancement has been demonstrated before to enable bi-exponential analysis of NMR decay curves from different tissue types in plants measured with functional MRI (Scheenen et al. 2002). Because of the unavoidable compromise between temporal and spatial resolution of MR imaging on its own, there always will be intrapixel and interpixel heterogeneity leading to averaging of properties to some extent. Also compared to the nonimaging approach, there are some disadvantages. First of all, imaging gradients can interfere with the diffusion encoding gradients, which has to be accounted for in the data analysis. Also the imaging approach decreases the time resolution related both to components with short $T_{2}$ values that will not be visible and to fast changes of the parameters under study that result from changing external conditions such as abiotic stress.

The presented method can be applied to a wide range of various porous systems for quantitative mapping of structural parameters. In the case of biomedical applications, more complicated models are necessary to take into account the significant contribution of extracellular water.

Pixel-by-pixel analysis (Fig. 8) yields detailed spatially resolved information on the size of restrictions (cell size) and interconnectivity of structure (permeability of intercellular pathways). Even small inhomogeneities in permeability can be distinguished. This level of information is lost in any nonimaging approach. The uncertainty of the calculated results, however, is increased due to the poor SNR of the individual signal attenuation plots. These data can still deliver spatially resolved high quality values by summing the SAP of individual pixels in an ROI selected on the basis of other parameter maps or images to improve the accuracy of the ADC values. As a result, the permeability and cell size are averaged characteristics of the selected ROI. The spatially resolved information content still exceeds nonspatially resolved approaches. Nonspatially resolved $T_{2}$-D correlation measurements, for example, where different fractions were discriminated on the basis of their $T_{2}$ values, will not provide the same detailed information. Selection of the ROIs is a crucial step because different tissue types can be mixed in one ROI on the basis of a wrong selection criterion. In this study different parameter maps and weighted images were used to enable optimal differentiation of tissue types.

Dependent on the age of the plant, the mean cell diameter in the outer phloem $(<5 \mathrm{~mm}$ from the periderm) of a carrot taproot was found to increase from $26.8 \mu \mathrm{m}$ at 77 days to $47.9 \mu \mathrm{m}$ at 171 days (determined by electron microscopy) (McGarry 1995). This region corresponds to the "phloem" and "cortex" region in our ROI analysis. The mean cell size (diameter) in phloem tissue (section 4 in Fig. 4a) was found to be $36 \pm 2 \mu \mathrm{m}$ (longitudinal) and $40 \pm 2 \mu \mathrm{m}$ (transverse); the cortex tissue (section 3 in Fig. 4a) was $37 \pm 10 \mu \mathrm{m}$ (longitudinal) and $43 \pm 3 \mu \mathrm{m}$ (transverse). These values correspond well with the microscopy data.

Given the pixel size $\left(625 \times 625 \times 3,000 \mu \mathrm{m}^{3}\right)$ for the time-dependent diffusion measurements and the fact that phloem and xylem tissue in particular is inhomogeneous by default, consisting of cells of different types, the approach of describing the data by one set of parameters is a simplification. Even the assumption that the fitted parameters represent average properties of the tissue in a pixel could be proven wrong. As a result of $T_{1}$ weighting, a diffusion 

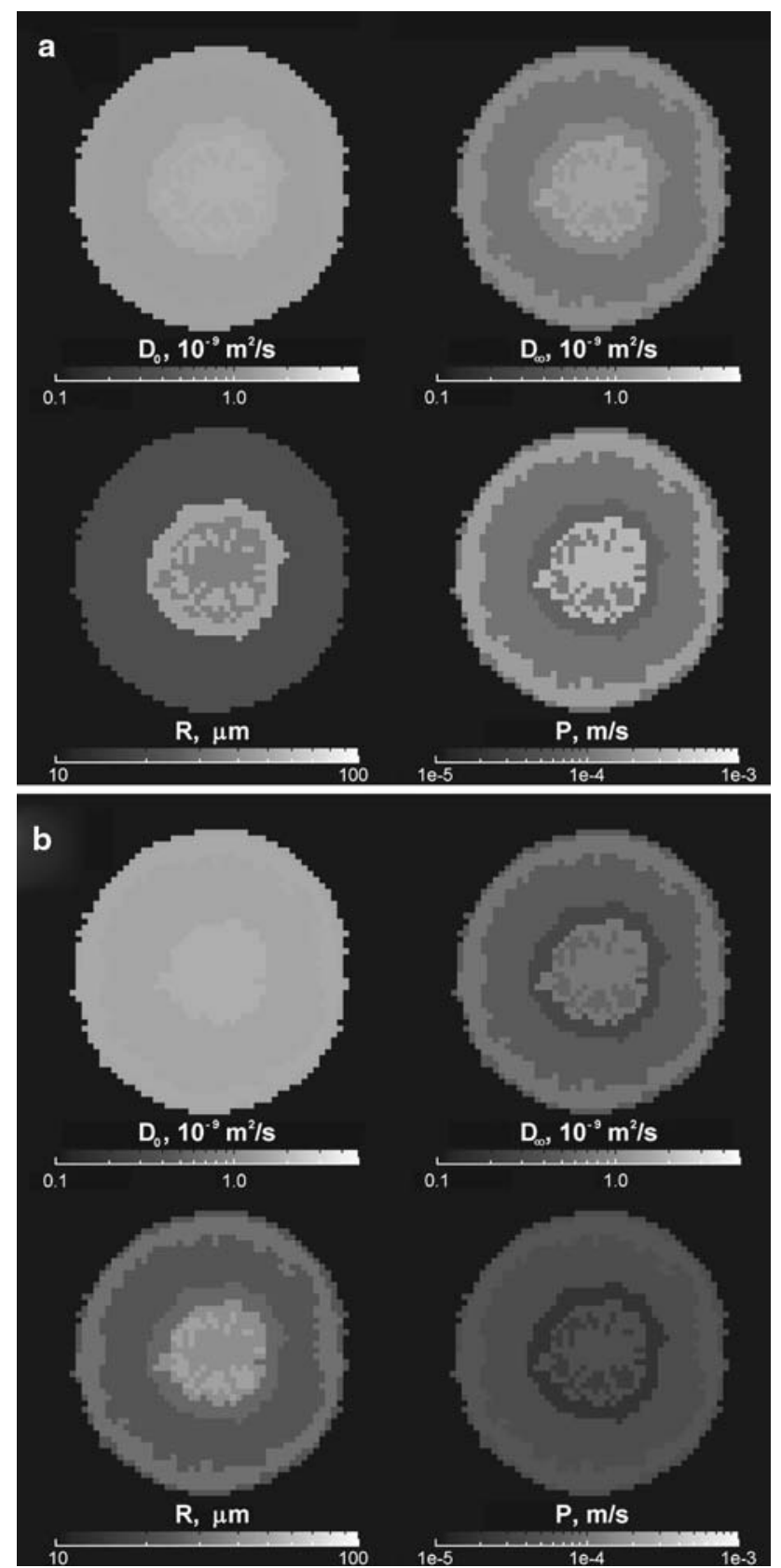

Fig. 7 Parametric maps of average ROI properties of carrot root (Fig. 4). Bulk and infinite diffusion coefficients $D_{0}$ and $D_{\infty}$ and cell size $R$ as a result of fitting of time-dependent ADC (Fig. 4) with Eq. 4 and calculated permeability $P$. Results of the diffusion experiment for axial (a) and in-plane (b) directions

constant measured at longer diffusion times will be dominated by contributions from large cells. On a cellular level, water in the vacuoles will dominate the result for the same reason. So the $D\left(t_{\mathrm{d}}\right)$ dependence may be distorted. However, in the case of a mature carrot taproot, the phloem and the xylem generally are mainly parenchymal cells that are nearly uniform in size and shape. So the presence of specialized cells in these tissues can be neglected to some extent.
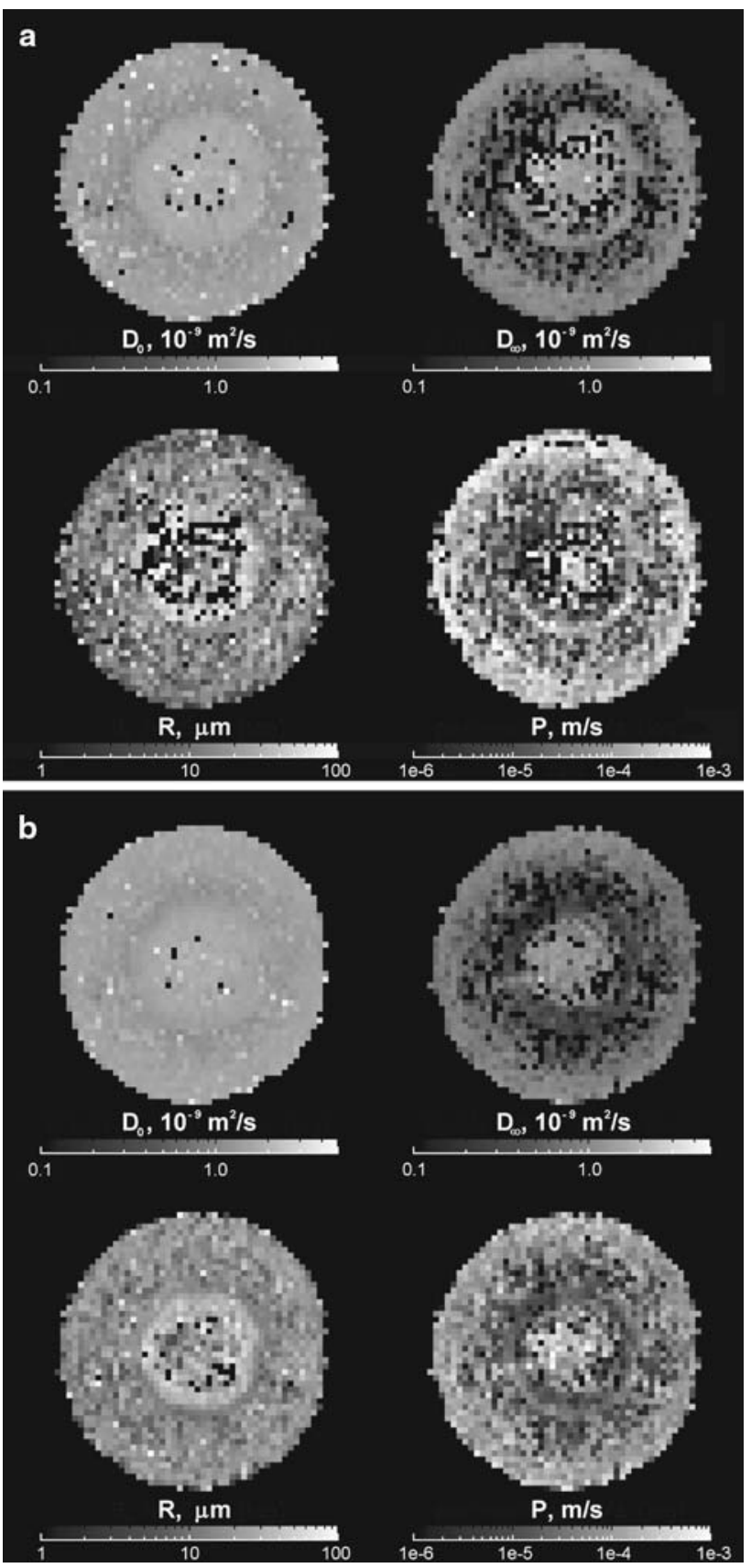

Fig. 8 Parametric maps of bulk and infinite diffusion coefficients $D_{0}$ and $D_{\infty}$ and cell size $R$ as a result of pixel-by-pixel analysis of timedependent ADC (Fig. 5) with Eq. 4 and calculated permeability $P$. Results of diffusion experiments for axial (a) and in-plane (b) directions

One can hope to solve this inhomogeneity problem by increasing the spatial resolution to reach the size of homogeneous regions or, as an alternative, by performing $T_{2}-D$ correlation measurements to separate the different fractions on the basis of their $T_{2}$ values and then applying the proposed pixel-by-pixel method for each $T_{2}$ fraction of the pixel. The time penalty for both approaches, however, 

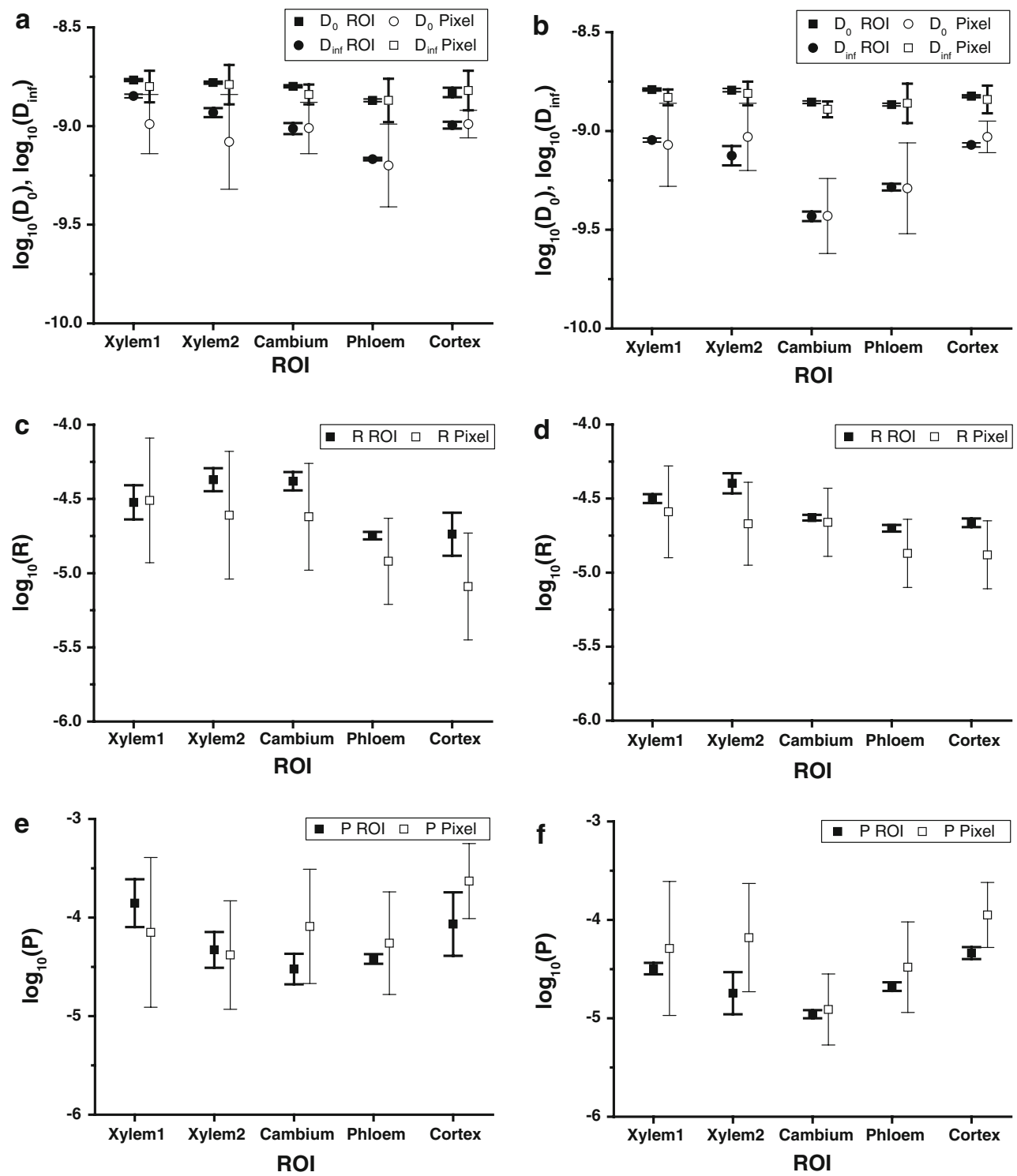

Fig. 9 Comparison of results of ROI analysis with error of the fit (closed symbols) versus mean amplitude-weighted decimal logarithm of parameters over the pixels of the same ROI as a result of pixel-bypixel fitting with standard deviation of the mean (open symbols). a

is very large and, based on the required time resolution for the process of interest, unacceptable for most studies of biological materials.

For plant tissues it is well known that signal intensity can be affected by susceptibility artifacts due to air inside the plant tissue, especially in the phloem and xylem tissue (sections 1, 2, and 4 in Fig. 4a). It has been shown that PFG-STE-based sequences for diffusion measurements appear to be "surprisingly" sensitive to internal field gradients in plant-based materials (Donker et al. 1997; Nestle et al. 2002). This needs extra attention and offers possibilities for improvement, for instance replacing the PFGSTE sequence with a so-called 13-interval sequence or

and $\mathbf{b} D_{0}$ (square) and $D_{\infty}$ (circle). $\mathbf{c}$ and $\mathbf{d}$ Radius $R$ of the cell. e and f Permeability $P$. a, c and e Slice (longitudinal) direction. b, $\mathbf{d}$ and $\mathbf{f}$ Readout (transverse) direction

modern varieties (Cotts et al. 1989; Galvosas et al. 2004). However, this is not essential for the proposed approach.

Open Access This article is distributed under the terms of the Creative Commons Attribution Noncommercial License which permits any noncommercial use, distribution, and reproduction in any medium, provided the original author(s) and source are credited.

\section{References}

Anisimov AV, Sorokina NY, Dautova NR (1998) Water diffusion in biological porous systems: a NMR approach. Magn Reson Imaging 16:565-568 
Boujraf S, Luypaert R, Eisendrath H, Osteaux M (2001) Echo planar magnetic resonance imaging of anisotropic diffusion in asparagus stems. Magn Reson Mater Phys Biol Med 13:82-90

Brownstein KR, Tarr CE (1979) Importance of classical diffusion in NMR studies of water in biological cells. Phys Rev A 19:2446-2453

Cho CH, Hong YS, Kang K, Volkov VI, Skirda V, Lee CYJ, Lee CH (2003) Water self-diffusion in Chlorella sp. studied by pulse field gradient NMR. Magn Reson Imaging 21:1009-1017

Codd SL, Seymour JD (2008) Magnetic resonance microscopy: spatially resolved NMR techniques and applications. Wiley$\mathrm{VCH}$, Weinheim

Conlon T, Outhred R (1972) Water diffusion permeability of erythrocytes using an NMR technique. Biochim Biophys Acta 288:354-361

Cotts RM, Hoch MJR, Sun T, Markert JT (1989) Pulsed field gradient stimulated echo methods for improved NMR diffusion measurements in heterogeneous systems. J Magn Reson 83:252-266

Crick F (1970) Diffusion in embryogenesis. Nature 225:420-422

Donker HCW, Van As H, Snijder HJ, Edzes HT (1997) Quantitative ${ }^{1} \mathrm{H}$-imaging of water in white button mushrooms (Agaricus bisporus). Magn Reson Imaging 15:113-121

Edzes HT, van Dusschoten D, Van As H (1998) Quantitative T-2 imaging of plant tissues by means of multi-echo MRI microscopy. Magn Reson Imaging 16:185-196

Galvosas P, Stallmach F, Karger J (2004) Background gradient suppression in stimulated echo NMR diffusion studies using magic pulsed field gradient ratios. J Magn Reson 166:164-173

Grebenkov DS (2007) NMR survey of reflected Brownian motion. Rev Mod Phys 79:1077-1137

Hong YS, Suh KJ, Yoon SW, Skirda V, Volkov V, Lee CH (2004) Effect of heat treatment on water permeability of yeast cells as measured by pulsed field gradient (PFG) NMR spectroscopy. Food Sci Biotechnol 13:586-590

Hong YS, Hong KS, Lee ES, Cho JH, Lee C, Cheong C, Lee $\mathrm{CH}$ (2009) MR imaging and diffusion studies of soaked rice. Food Res Int 42:237-245

Ionenko IF, Anisimov AV, Karimova FG (2006) Water transport in maize roots under the influence of mercuric chloride and water stress: a role of water channels. Biol Plant 50:74-80

Kärger J (1971) Der Einfluß der Zweibereichdiffusion auf die Spinechodämpfung unter Berücksichtigung der Relaxation bei Messungen mit der Methode der gepulsten Feldgradienten. Ann Phys 27:107-109

Kärger J, Pfeiffer H, Heink W (1988) Principles and application of self-diffusion measurements by nuclear magnetic resonance. Adv Magn Reson 12:1-89

Kuchenbrod E, Haase A, Benkert R, Schneider H, Zimmermann U (1995) Quantitative NMR microscopy on intact plants. Magn Reson Imaging 13:447-455

Latour LL, Svoboda K, Mitra PP, Sotak CH (1994) Time-dependent diffusion of water in a biological model system. Proc Natl Acad Sci USA 91:1229-1233

McGarry A (1995) Cellular basis of tissue toughness in carrot (Daucus Carota L.) storage roots. Ann Bot 75:157-163

Mitra PP, Sen PN, Schwartz LM (1993) Short-time behavior of the diffusion-coefficient as a geometrical probe of porous-media. Phys Rev B 47:8565-8574

Neeman M, Freyer JP, Sillerud LO (1990) Effects of the imaging gradients on the determination of diffusion coefficients. J Magn Reson 90:303-312

Nestle N, Qadan A, Galvosas P, Suss W, Kärger J (2002) PFG NMR and internal magnetic field gradients in plant-based materials. Magn Reson Imaging 20:567-573

Norris DG (2001) The effects of microscopic tissue parameters on the diffusion weighted magnetic resonance imaging experiment. NMR Biomed 14:77-93
Paran Y, Bendel P, Margalit R, Degani H (2004) Water diffusion in the different microenvironments of breast cancer. NMR Biomed $17: 170-180$

Pfeuffer J, Dreher W, Sykova E, Leibfritz D (1998a) Water signal attenuation in diffusion-weighted H-1 NMR experiments during cerebral ischemia: influence of intracellular restrictions, extracellular tortuosity, and exchange. Magn Reson Imaging 16:1023-1032

Pfeuffer J, Flogel U, Leibfritz D (1998b) Monitoring of cell volume and water exchange time in perfused cells by diffusion-weighted H-1 NMR spectroscopy. NMR Biomed 11:11-18

Regan DG, Kuchel PW (2000) Mean residence time of molecules diffusing in a cell bounded by a semi-permeable membrane: Monte Carlo simulations and an expression relating membrane transition probability to permeability. Eur Biophys J 29:221-227

Scheenen TWJ, van Dusschoten D, de Jager PA, Van As H (2000) Microscopic displacement imaging with pulsed field gradient turbo spin-echo NMR. J Magn Reson 142:207-215

Scheenen TWJ, Vergeldt FJ, Windt CW, de Jager PA, Van As H (2001) Microscopic imaging of slow flow and diffusion: a pulsed field gradient stimulated echo sequence combined with turbo spin echo imaging. J Magn Reson 151:94-100

Scheenen T, Heemskerk A, de Jager A, Vergeldt F, Van As H (2002) Functional imaging of plants: a nuclear magnetic resonance study of a cucumber plant. Biophys J 82:481-492

Sen PN (2003) Time-dependent diffusion coefficient as a probe of the permeability of the pore wall. J Chem Phys 119:9871-9876

Sen PN (2004) Time-dependent diffusion coefficient as a probe of geometry. Concepts Magn Reson Part A 23A:1-21

Sibgatullin TA, Anisimov AV, de Jager PA, Vergeldt FJ, Gerkema E, Van As H (2007) Analysis of diffusion and relaxation behavior of water in apple parenchymal cells. Biophysics 52:268-276

Snaar JEM, Van As H (1992) Probing water compartment and membrane permeability in plant cells by proton NMR relaxation measurements. Biophys J 63:1654-1658

Stejskal EO, Tanner JE (1965) Spin diffusion measurements: spin echoes in the presence of a time-dependent field gradient. J Chem Phys 42:288-292

Steudle E (2001) The cohesion-tension mechanism and the acquisition of water by plant roots. Annu Rev Plant Physiol Plant Mol Biol 52:847-875

Suh KJ, Hong YS, Skirda VD, Volkov VI, Lee CY, Lee CH (2003) Water self-diffusion behavior in yeast cells studied by pulsed field gradient NMR. Biophys Chem 104:121-130

Tanner JE (1978) Transient diffusion in a system partitioned by permeable barriers. Application to NMR measurements with a pulsed field gradient. J Chem Phys 69:1748-1754

Valiullin R, Skirda V (2001) Time dependent self-diffusion coefficient of molecules in porous media. J Chem Phys 114:452-458

Valiullin RR, Skirda VD, Stapf S, Kimmich R (1997) Molecular exchange processes in partially filled porous glass as seen with NMR diffusometry. Phys Rev E 55:2664-2671

Van As H (2007) Intact plant MRI for the study of cell water relations, membrane permeability, cell-to-cell and long distance water transport. J Exp Bot 58:743-756

van der Weerd L (2002) Magnetic resonance imaging of plants: plant water status and drought stress response. $\mathrm{PhD}$ Thesis, Wageningen University

van der Weerd L, Claessens MMAE, Ruttink T, Vergeldt FJ, Schaafsma TJ, Van As H (2001) Quantitative NMR microscopy of osmotic stress responses in maize and pearl millet. J Exp Bot 52:2333-2343

van der Weerd L, Claessens MMAE, Efde C, Van As H (2002a) Nuclear magnetic resonance imaging of membrane permeability changes in plants during osmotic stress. Plant Cell Environ 25:1539-1549 
van der Weerd L, Melnikov SM, Vergeldt FJ, Novikov EG, Van As H (2002b) Modelling of self-diffusion and relaxation time NMR in multicompartment systems with cylindrical geometry. J Magn Reson 156:213-221

Waldeck AR, Nouri Sorkhabi MH, Sullivan DR, Kuchel PW (1995) Effects of cholesterol on transmembrane water diffusion in human erythrocytes measured using pulsed field gradient NMR. Biophys Chem 55:197-208

Zakhartchenko NL, Skirda VD, Valiullin RR (1998) Self-diffusion of water and oil in peanuts investigated by PFG NMR. Magn Reson Imaging 16:583-586 